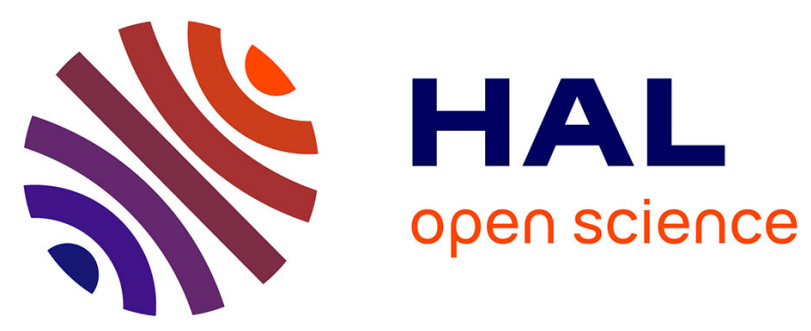

\title{
A qualitative numerical analysis of rotor-casing interactions in centrifugal compressors of helicopter engines
}

Markus B Meingast, Alain Batailly, Mathias Legrand, Jean-Philippe Ousty

\section{- To cite this version:}

Markus B Meingast, Alain Batailly, Mathias Legrand, Jean-Philippe Ousty. A qualitative numerical analysis of rotor-casing interactions in centrifugal compressors of helicopter engines. ASME 2013 International Design Engineering Technical Conferences and Computers and Information in Engineering Conference, Aug 2013, Portland, United States. 10.1115/DETC2013-12212 . hal-00851397

\section{HAL Id: hal-00851397 \\ https://hal.science/hal-00851397}

Submitted on 20 Aug 2013

HAL is a multi-disciplinary open access archive for the deposit and dissemination of scientific research documents, whether they are published or not. The documents may come from teaching and research institutions in France or abroad, or from public or private research centers.
L'archive ouverte pluridisciplinaire HAL, est destinée au dépôt et à la diffusion de documents scientifiques de niveau recherche, publiés ou non, émanant des établissements d'enseignement et de recherche français ou étrangers, des laboratoires publics ou privés. 


\title{
Analyse numérique qualitative d'interactions rotor/stator dans un compresseur haute pression d'un moteur d'hélicoptère
}

M. Meingast, Laboratoire de dynamique des structures et vibrations, université McGill, Québec A. Batailly, Laboratoire de dynamique des structures et vibrations, université McGill, Québec M. Legrand, Laboratoire de dynamique des structures et vibrations, université McGill, Québec J.-P. Ousty, Turbomeca, France

\section{Résumé}

Lors de l'étude numérique ou expérimentale d'interactions rotor/stator générées par du contact unilatéral ou du frottement, une grande variété de phénomènes complexes peuvent se produire. Bien que l'essentiel du contenu des réponses structurelles puissent être analysées dans le cadre d'un formalisme linéaire simplifié, la nature hautement non-linéaire du contact génère des signaux complexes qui doivent être minutieusement analysés. Le travail présenté dans cet article porte sur l'analyse de ce type de simulations et les investigations envisageables en ayant pour but de fournir une méthodologie systématique d'analyse. Les simulations d'interaction sont réalisées sur un compresseur centrifuge d'un moteur d'hélicoptère moderne pour lequel il est supposé que le carter est ovalisé, c'est-à-dire déformé selon un mode à deux diamètres. Ce travail se focalise sur des aspects géométriques tels que la symétrie cyclique et le phénomène d'aliasing des régimes moteurs sur certains diamètres nodaux ainsi que sur des aspects typiquement non-linéaires comme les sous- et super-harmoniques observées dans la réponse de la structure dans le but de caractériser les propriétés dynamiques de l'interaction. Les résultats sont présentés en utilisant une transformée de Fourier en deux dimensions (temps et espace) de la réponse prédite. Des pics principaux sont observés le long du régime moteur correspondant à la fréquence principale du contact imposée par la forme ovalisée du carter. Il est notamment observé que certains diamètres nodaux répondent de façon non négligeable du fait de la combinaison de l'aliasing et de super-harmoniques du chargement imposé.

\section{A qualitative numerical analysis of rotor-casing interactions in centrifugal compressors of helicopter engines}

\begin{abstract}
In experimental and numerical investigations of unilateral contact and friction induced rotorcasing interactions, a variety of complex phenomena is expected. Although most of the features of the structural responses can be explained within a simplifying linear framework, the nonlinear nature of contact and friction forces induce complicated responses which require an appropriate methodology to be conveniently analyzed. The presented work focuses on a thorough numerical exploration of such undesired events in an attempt to provide a dedicated systematic method of analysis.

Interaction simulations are carried out on the centrifugal compressor of a modern helicopter engine, for which it is assumed that the casing is rigidly distorted along a mathematical shape exhibiting distinct nodal diameters. The proposed work focuses on linear geometric aspects, such as cyclic symmetry and spatial aliasing of engine orders on nodal diameters as well as nonlinear attributes, such as sub-and super-harmonic participations in the response, in order to properly characterize the dynamics of the interaction.

The results are presented as space and time two-dimensional Fourier transforms of the numerically predicted response. Dominant responses are visible along time harmonics of the forcing frequency imposed by the assumed shape of the casing. It is observed that the participation of some nodal diameters in the response is a consequence of both the aliasing effect and super-harmonic forcing terms.
\end{abstract}




\section{INTRODUCTION}

Helicopter and airplane engines feature small operating clearances between rotating and stationary components in order to avoid aerodynamic leaks that may arise in the low-pressure and high-pressure compressors. In this context, extreme operating conditions may lead to the consumption of this clearance and corresponding unilateral contact and friction occurrences between the structures. Abradable liners that are commonly deposited along the circumference of the casing are known to be robust solutions that mitigate the level of vibration of the contacting structures. However, undesired interaction phenomena in normal operating conditions were observed in axial compressors [1] and a better understanding of such events has become a key challenge for engine manufacturers.

In this area, recent numerical developments focused on a single blade of axial compressors[2, 3]. There is now the need for a general methodology relevant to a large family of structures featuring cyclic symmetry for which such interaction occurrences may arise. For instance, as opposed to axial compressors, centrifugal compressors or automotive turbochargers feature an intricate blade-tip geometry which necessitates a very accurate description of the contact configuration. The goal of this contribution is to collect the concepts of practical interest needed for a systematic analyzis of heavy-duty time-marching simulations of cyclic structures experiencing rigid casing contact interactions.

As a case study, the centrifugal compressor, also named impeller, of a helicopter engine is considered. Literature pertaining to the vibration analysis of impellers, and to a larger extent within contact mechanics, is fairly limited. The only available studies deal with modal responses and are detailled in $[4,5,6,7]$. Recently, the geometry of interest in the presented case study was analyzed in [8]. Based on an existing numerical strategy [3], various numerical simulations are conducted to explore two distinct contact scenarios involving the impeller and a surrounding rigid casing. Meticulous attention is paid to expected super and sub-harmonic resonances as well as the aliasing effect.

The first section of the paper deals with the presentation of the systems of interest. Details on the theoretical background and associated numerical considerations are then provided in the second section. The last section of the paper is related to an application on a rotor test case.

\section{GENERAL CONTEXT}

Simulations of structural interactions through unilateral contact and friction occurrences between rotating structures featuring cyclic symmetry and their surrounding casings featuring axial symmetry are introduced.

\section{Investigated system}

A schematic of the system of interest is displayed in Fig. 1. The cyclic symmetric structure is assumed to be clamped on a perfectly rigid shaft. The structure is divided into $\mathrm{N}$ elementary sectors numbered $i-1, i$, and $i+1$ in Fig. 1 . On each sector, dedicated boundary nodes forming the contact interface are defined to manage contact conditions. These nodes are typically located along the tip of a blade, but may also lie on a blade shroud for instance. The rotational speed is $\Omega$.

Contact is initiated through an enforced casing distortion. As usually assumed, the casing is deformed along one of its free vibration modes which exhibits nodal diameters: accordingly, as depicted in Fig. 2, a given number of areas in which the tip gap is consumed are evenly distributed along the circumference of the casing ( $\quad$ ). In the sequel, the casing deformation is characterized by its number of lobes $\mathrm{N}_{l}$.

This article is an extension of the methodology presented in [3], for a single sector of an axial compressor, to any cyclic symmetric structure such as axial compressor, centrifugal 


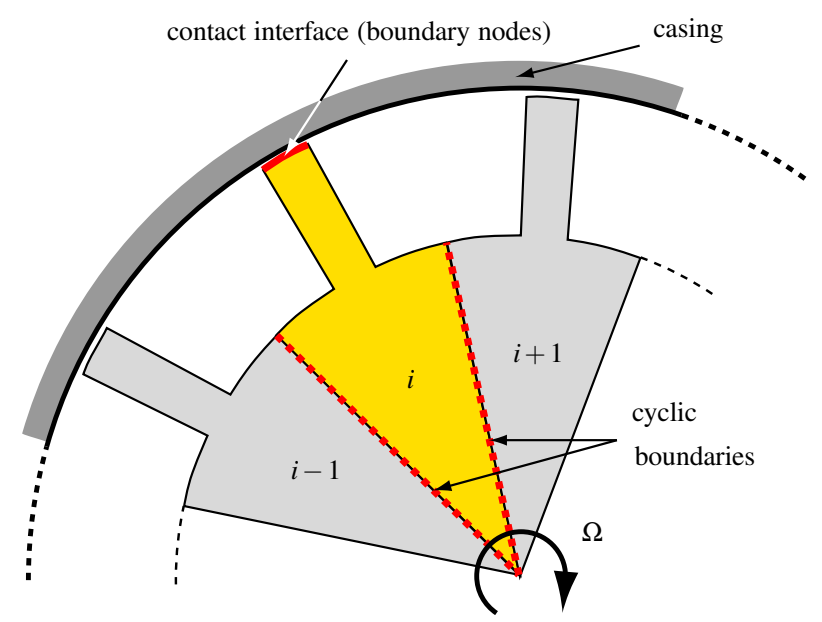

Figure 1 - PARTIAL REPRESENTATION OF THE SYSTEM.
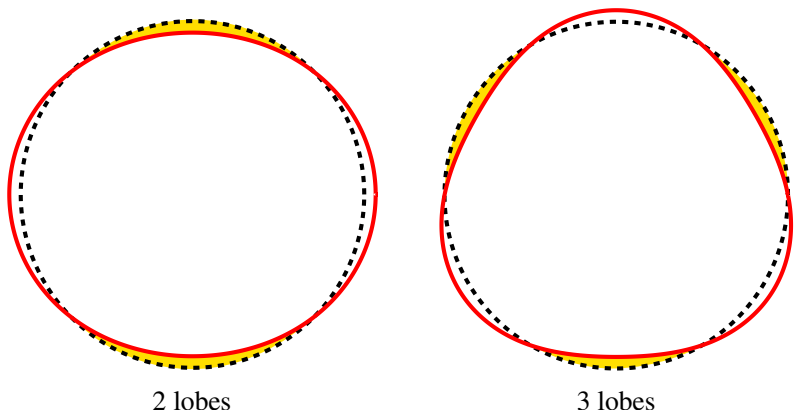

Figure 2 - CASING DISTORTIONS.

compressor or turbocharger. The assumption of a pefectly rigid ${ }^{1}$ casing is made in order to reduce computation times. The validity of such hypothesis may be questioned depending on the type and the scale of the involved structures ${ }^{2}$. Considering a flexible casing is possible with the proposed strategy and work is in progress so that the proposed strategy may be applied, for example, to the detection of modal interaction behaviours between two flexible structures.

\section{Quantities of interest}

Vibratory levels as well as local stresses within the blades are crucial quantities when designing the stage of a turbomachine. For that reason, the proposed methodology relies on precise 3D finite element models. When contact with the casing is accounted for, maximum vibration level evolves nonlinearly with the rotational speed and critical speeds - for which the vibratory level reaches local maximum values - may thus be defined. In order to identify these speeds, a three-step procedure in the frequency domain is proposed:

1. A contact configuration is defined with $\mathrm{N}_{l}$ lobes and a specific rotational speed range is considered $\Omega \in\left[\Omega_{\min } ; \Omega_{\max }\right]$.

2. For each $\Omega$, time-marching contact simulations are carried out until a steady state is reached ${ }^{3}$;

3. Fourier transforms of the radial displacement of the leading edge of the blade are computed for each $\Omega$ and organized in a waterfall diagram as pictured in Fig. 3(a); Comple-

${ }^{1}$ While globally deformed, the casing is considered perfectly rigid, meaning it is insensitive to the contacts with the rotating structure.

${ }^{2}$ The assumption of a perfectly rigid casing is frequently made in the case of large axial compressors.

${ }^{3}$ It is assumed that steady state within this framework does not necessarily imply a purely periodic solution, but rather a solution of stable amplitude. 
mentary information in the form of color maps as shown in Fig. 3(b) is also supplied.

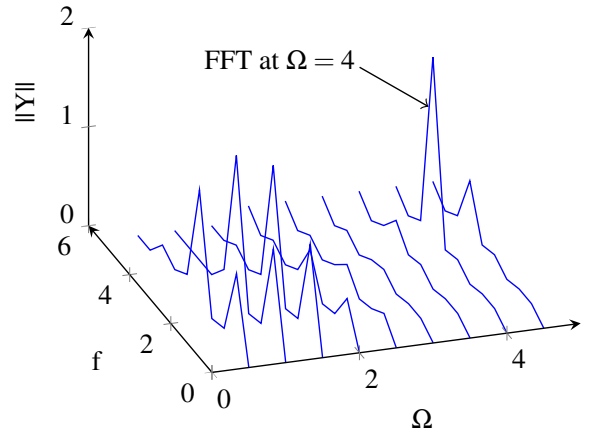

(a) waterfall diagram...

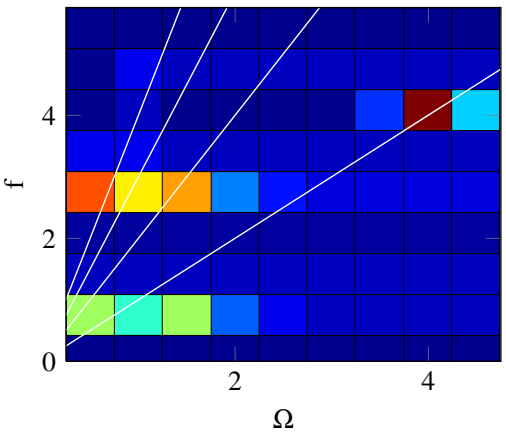

(b) ... and corresponding interaction map with color scale

Figure 3 - FREQUENCY DOMAIN RESULTS

An engine order (EO) is defined as the number of excitation cycles per revolution of the rotating assembly. The corresponding EO lines $f=k \Omega$ with $k=1,2,3,4$ are displayed in the interaction map as white lines and bring useful insight of the observed interaction phenomena. In particular, highest vibrational levels are expected along these lines once steady state is reached. In the following, the EO lines are depicted as black dashed lines for the sake of clarity.

\section{THEORETICAL BACKGROUND}

The proper analysis of the conducted time-marching contact simulations carried out in the remainder require a few key notions that are summarized in this section. The solution method is not recalled and the reader may refer to [3] for details.

\section{Cyclic symmetry}

The rotating bladed-disk assembly is perfectly tuned and is thus said to be cyclic symmetric. Such structures exhibit block-circulant mass $\mathbf{M}$ and stiffness $\mathbf{K}$ matrices in cylindrical coordinates:

$$
\mathbf{Y}=\mathbf{K} \text { or } \mathbf{M}=\left[\begin{array}{cccccc}
\mathbf{S} & \mathbf{C}^{\mathrm{T}} & \mathbf{0} & \mathbf{0} & \ldots & \mathbf{C} \\
\mathbf{C} & \mathbf{S} & \mathbf{C}^{\mathrm{T}} & \mathbf{0} & \ldots & \mathbf{0} \\
\mathbf{0} & \mathbf{C} & \mathbf{S} & \mathbf{C}^{\mathrm{T}} & \mathbf{0} & \ldots \\
\vdots & & & \ddots & & \\
\mathbf{0} & \ldots & \mathbf{0} & \mathbf{C} & \mathbf{S} & \mathbf{C}^{\mathrm{T}} \\
\mathbf{C}^{\mathrm{T}} & \mathbf{0} & \ldots & \mathbf{0} & \mathbf{C} & \mathbf{S}
\end{array}\right]
$$

where the blocks $\mathbf{S}$ and $\mathbf{C}$ are associated to a sector and to the coupling terms between two sectors, respectively. The number of rows in Eq. (1) reflects the number of sectors N. Block circulant matrices shall be block-diagonalized through a proper Fourier transform $\mathbf{F}$ [9]:

$$
\mathbf{F}^{\mathrm{T}} \mathbf{Y F}=\left[\begin{array}{ccccc}
\mathbf{B}_{0} & & & & \\
& \mathbf{B}_{1} & & (\mathbf{0}) & \\
& & \mathbf{B}_{2} & & \\
& (\mathbf{0}) & & \ddots & \\
& & & & \mathbf{B}_{\left\lfloor\frac{\mathrm{N}}{2}\right\rfloor}
\end{array}\right]
$$

Accordingly, the study of cyclic symmetric structures may be conducted as $\left\lfloor\frac{\mathrm{N}}{2}\right\rfloor+1$ uncoupled problems. Each block $\mathbf{B}_{i}$ refers to a spatial harmonic of the structure, also named nodal 
diameter and denoted $n_{d}$ in the following [9]. For instance, in the case study considered below, the impeller possesses $\mathrm{N}=10$ sectors and hence $\mathrm{N}_{h}=6$ distinct spatial harmonics $\mathbf{B}_{i}$, $i=0, \ldots, 5$. The terminology nodal diameter is here preferred to spatial harmonic in order to avoid possible confusion with time harmonics defined below. For a detailed discussion on cyclic symmetry and nodal diameters, see [10].

The free vibration modes of a cyclic symmetric structure are clustered in modal families [9]. Each modal family of $\mathrm{N}$ modes refers to a specific type of vibration of the blades (bending, torsion, disk modes...) across all possible nodal diameters: the first single mode with $n_{d}=0$ is a stationary wave while the higher modes come as orthogonal pairs involving $n_{d}=1$ to $n_{d}=\mathrm{N}_{h}-2$ nodal diameters that can be combined into travelling waves. For an even number of sectors, the last mode features $n_{d}=\mathrm{N}_{h}-1$ nodal diameters will appear as a single stationary mode for a even number of sectors, or as a dual mode if the number of sectors is odd.

\section{Resonance of a system with contact}

Due to unilateral contact, the system is nonlinear and nonsmooth. In addition to fundamental harmonic resonances that usually characterize linear systems, nonlinear systems are known to feature sub-and super-harmonic resonances as explained in [11] for a single-DOF system. A wide body of work is available concerning the sub-harmonic resonances oscillators with impact $[12,13,14]$. Recent references may also be found dealing with the identification of sub-harmonic resonances in a large variety of industrial applications [15]. Yet, in the framework of nonlinear nonsmooth cyclic symmetric contact problems, sub- and superharmonics are not understood very well. Sub- and super-harmonic resonances are generally defined as follows:

1. A super-harmonic response of order $n$ is a periodic solution having the same period as that of the external excitation $\omega$ featuring a significant Fourier component for $f=n \omega$.

2. A sub-harmonic means a periodic solution having the same period as that of the external excitation $\omega$ featuring a significant Fourier component for $f=\frac{n}{p} \omega$ with $n$ and $p$ integers and $p>n$.

\section{Aliasing effect}

The aliasing effect is well known for forced response problems in turbomachinery aeroelasticity. Due to the finite number of blades and hence possible nodal diameters, high EO excitations respond on spatial aliases on the rotor. For a generalized linear forcing, e.g. due to upstream flow perturbation from the flow coming off of a vane stage, the relationship follows (3). A graphic representation is depicted in Fig.4 for the previously mentioned industrial application. If for example three vanes are positioned upstream of the impeller, three equally distributed flow disturbances are encountered by each blade during one revolution. Assuming the flow disturbance can be modeled as a simple harmonic spatial excitation, it is to be considered of $\mathrm{EO}=3$. The response will therefore follow a $n_{d}=3$ pattern. Generally, the forcing contains a fundamental harmonic as well as higher harmonics, e.g. the forcing can be modeled by a finite Fourier series. The first harmonic of the aforementioned flow disturbance pattern, corresponds to a $\mathrm{EO}=6$ excitation (i.e. twice of the fundamental excitation). Due to the limited number of nodal diameters, the $\mathrm{EO}=6$ excitation responds in a $n_{d}=4$ pattern on the bladed disk.

The same assumptions hold for the excitation of the bladed disk due to casing deformation initiated contact interactions. For a 3-lobe casing deformation $\left(\mathrm{N}_{l}=3\right)$ the dominant contact pattern is along $\mathrm{EO}=3$, and hence the response is dominant on $n_{d}=3$, see (-) in Fig. 4. Due to the nonlinearity of contact, the excitation will also feature higher EO content. Higher spatial harmonics of $\mathrm{EO}=6,9,12, \ldots$ will respond on their spatial aliases $n_{d}=4,1,2, \ldots$, see (- - ) in Fig. 4. 


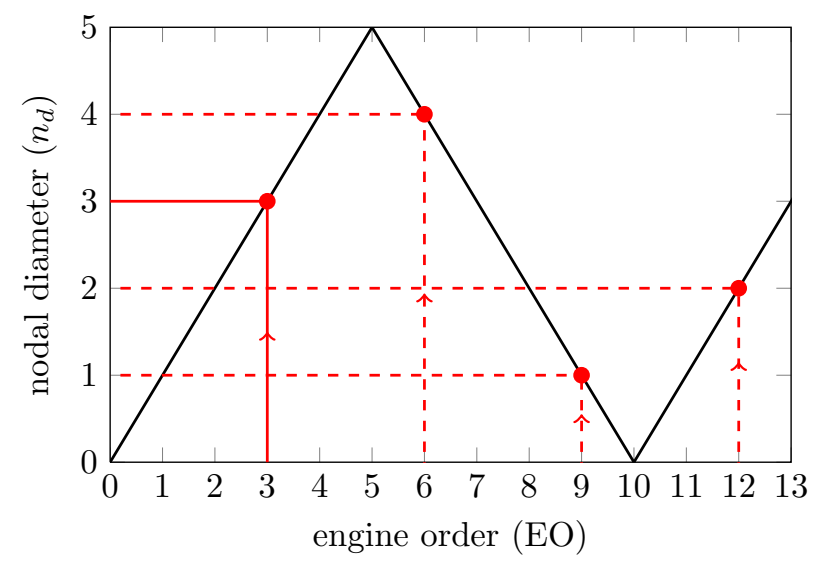

Figure 4 - ALIASING EFFECT: PREDICTING THE NUMBER OF NODAL DIAMETERS ASSOCIATED WITH AN ENGINE ORDER: A THREE-LOBE FUNDAMENTAL EO (-) AND CORRESPONDING HIGHER HARMONICS (- - - ).

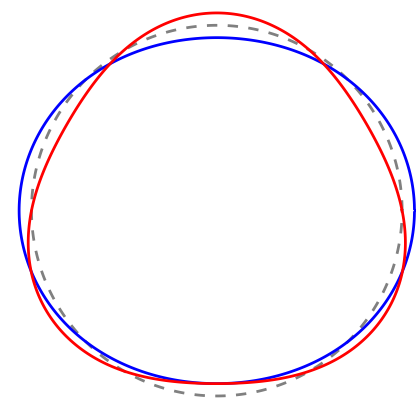

(a) Typical casing deformations $\mathrm{N}_{l}=2(-)$, $\mathrm{N}_{l}=3(-)$ on reference geometry $(---)$.

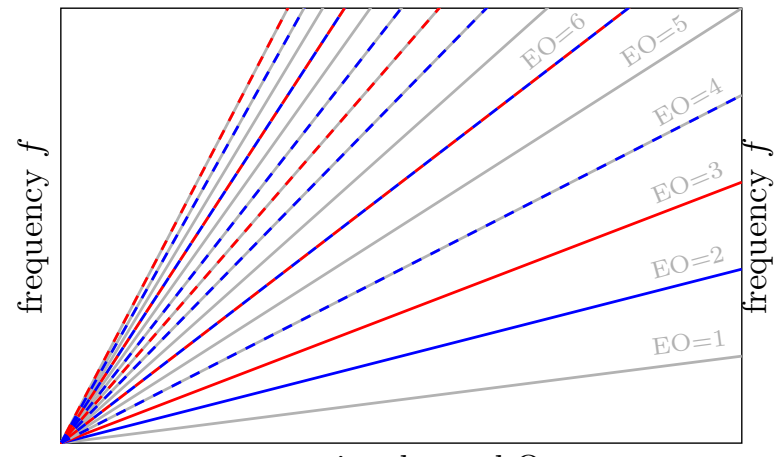

rotational speed $\Omega$

(c) Superharmonics: $\mathrm{EO}=n \mathrm{~N}_{l}$ with $n$ positive integer.

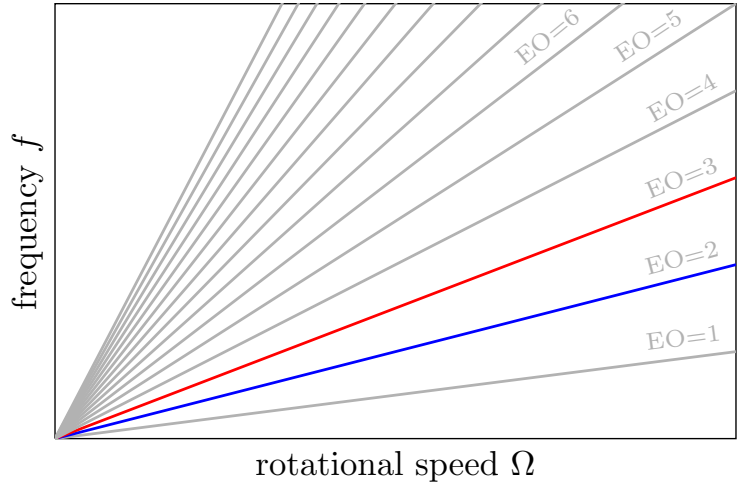

(b) Linear considerations: $\mathrm{EO}=\mathrm{N}_{l}$ only.

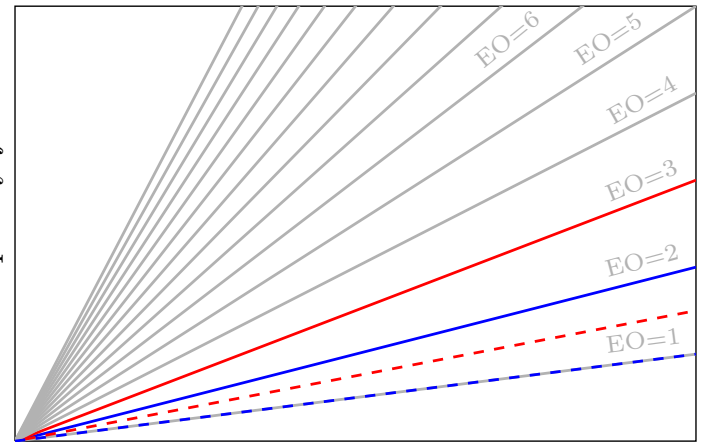

rotational speed $\Omega$

(d) Subharmonics: $\mathrm{EO}=\frac{n}{p} \mathrm{~N}_{l}$ with $n$ and $p$ positive integer and $p>n$.

Figure 5 - RESPONSE TO 2-LOBE AND 3-LOBE EXCITATIONS AND THEIR HARMONICS

$$
n_{d}= \begin{cases}\mathrm{EO}(\bmod \mathrm{N}) & \text { if } \mathrm{EO}(\bmod \mathrm{N}) \leq\left\lfloor\frac{\mathrm{N}}{2}\right\rfloor \\ \mathrm{N}-\mathrm{EO}(\bmod \mathrm{N}) & \text { if } \mathrm{EO}(\bmod \mathrm{N})>\left\lfloor\frac{\mathrm{N}}{2}\right\rfloor\end{cases}
$$

The same way the Nyquist frequency determines the maximal measurable temporal frequency to be half of the sampling frequency, which is here a spatial sampling frequency, the number of sectors on the bladed disk determines the highest nodal diameter that can respond. Visualizing the aliasing effect, one may consider the function $\cos (7 \theta)$ for $\theta \in[0 ; 2 \pi]$ in Fig. 6 . The 10 blades are evenly spaced and are represented by the discrete dots (o) located at 


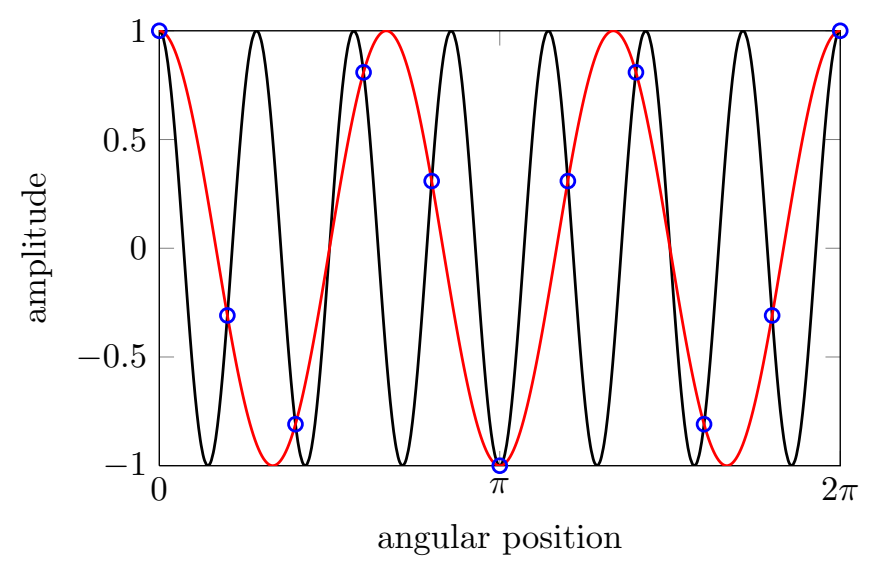

Figure 6 - ALIASING EFFECT.

$\theta=2 k \pi / 10, k=1, \ldots, 10$. As illustrated, $\cos (7 \theta)$ is regarded by the set of blades as the function $\cos (3 \theta)$. This physically means that a non negligible peak of resonance observed along EO 7 corresponds to a 3 nodal-diameter free vibration mode.

Figure 5, depicts two casing deformations and expected response EO, that are subsequently applied on the casing surrounding the impeller in the following section. The two casings are shown in 5(a) for a two-lobe (-) and three-lobe $(-)$ deformation. Under linear considerations, the response on the rotor is expected to be on $n_{d}=2$ and $n_{d}=3$ respectively, throughout the rotational speed range, see Fig. 5(b). Incorporating the nonlinearity due to contact, super- as well as sub-harmonic responses need to be considered. Figure 5(c) shows the fundamental responses EO (-) and (-) as well as their respective super-harmonic EO (-- -) and (- - ). Analog to the super-harmonic, sub-harmonic EO are depicted in Fig. 5(d).

\section{CASE STUDY}

Note: the term engine order (EO) refers to the harmonics as well as super- and sub-harmonics of the structural time response spectrum while nodal diameter refers to the spatial harmonics of a cyclic symmetric structure.

\section{Helicopter impeller}

The structure of interest is the helicopter engine impeller depicted in Fig. 7. The rotor consists of 10 elementary sectors, each containing one main blade and one splitter blade. To reduce the computational cost, contact is limited to the main blade tip chord. This assumption is consistent with the fact that the first free vibration modes of the impeller - which essentially involve the main blade and are also the modes featuring highest levels of strain energy - have lower eigenfrequencies than the free vibration modes involving the splitter blade. One contact interface is defined for each main blade through ten equally distributed nodes along the chord, see (๑) in Fig. 7.

\section{Contact configurations}

In order to better understand the notions introduced previously, the following two configurations are considered as pictured in Fig. 5(a):

Configuration 1 The casing is deformed along a 2-nodal diameter free vibration mode and features two symmetric privileged contact areas, $\mathrm{N}_{l}=2$.

Configuration 2 The casing is deformed along a 3-nodal diameter free vibration mode and features three symmetric privileged contact areas, $\mathrm{N}_{l}=3$. 


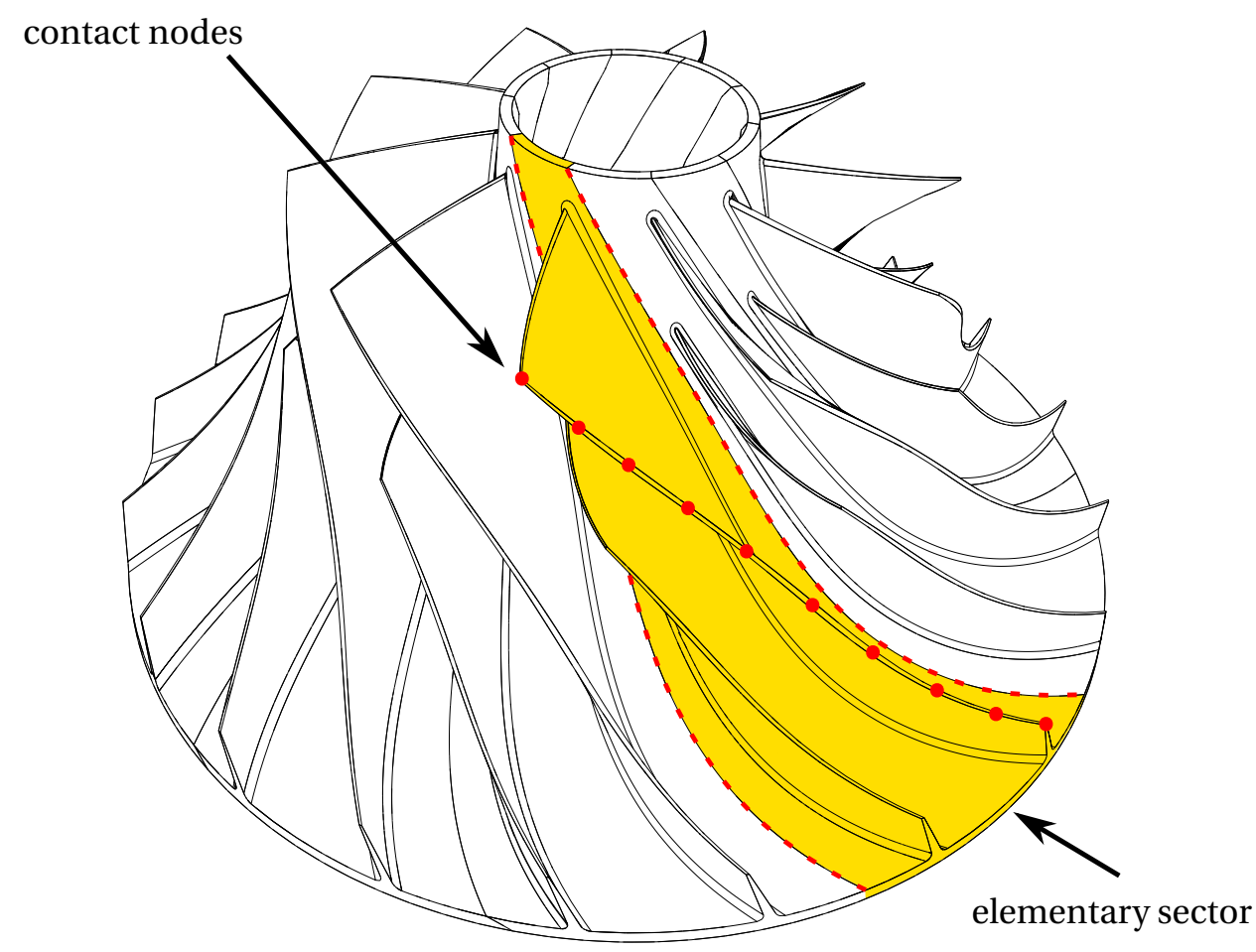

Figure 7 - HELICOPTER IMPELLER: CONTACT NODES (•), CYCLIC BOUNDARY (- - - ).

\begin{tabular}{ccc}
\hline & configuration 1 & configuration 2 \\
\hline $\mathrm{N}_{l}$ & 2 & 3 \\
$\mathrm{~N}_{h}$ & 6 & 6 \\
$\mathrm{~N}$ & 10 & 10 \\
$\Omega$ & {$[2.95 ; 5.45]$} & {$[2.95 ; 5.45]$} \\
\hline
\end{tabular}

Table 1 - SIMULATION PARAMETERS

Other parameters are summarized in Tab. 1. The casing restriction schematically follows the blade tip curvature as shown in Fig. 8 but the amplitude of the casing deformation is increased around the trailing edge where contact is thus initiated. In agreement with the number of lobes on the casing in each configuration, the main responses for both cases are expected to occur on EO 2 for configuration 1 and EO 3 for configuration 2. Because the system is highly nonlinear, super and sub-harmonic engine orders are also expected in agreement with what is depicted in Figs. 5(c) and 5(d).

\section{Results}

The results are shown for a wide rotational speed range $\Omega \in[2.95 ; 5.45]$ for each configuration. Interaction maps similar to the one in Fig. 3(b) with a higher resolution both in rotational

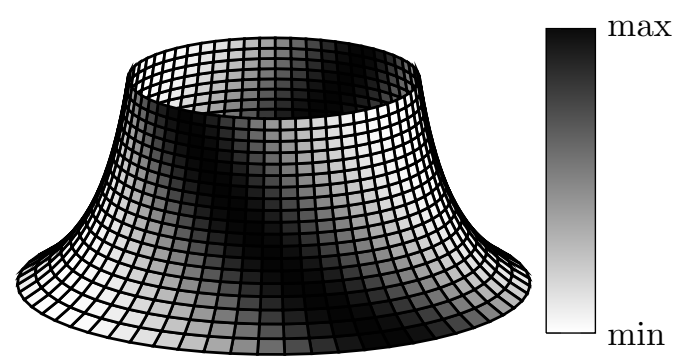

Figure 8 - CASING RESTRICTION FOR A 2-LOBE SETUP. 
speed and frequency are plotted along with several EO (black dashed lines) in Figs. 9 and 10.

To efficiently post-process the data acquired from the time domain simulations, a twodimensional Fourier transform is performed. The first dimension of the Fourier transform deals with the temporal frequency content of the response of the system, whereas the second dimension captures the spatial harmonic content of the response. Using this methodology allows for a clear and efficient analysis of the responding nodal diameters for a given contact setup, rotational speed and forcing harmonic.

\section{Configuration 1}

The interaction map given in Fig. 9(a) stands for the trailing edge of one sector of the impeller.

In agreement with the fact that $\mathrm{N}_{l}=2$, the first main response is visible on EO 2 marked as main EO line in Fig. 9(a). Further even EO lines show large amplitude responses, namely 4 th, 6 th, 8 th, etc. These are super-harmonics of the fundamental excitation frequency. Consistently with the even number of lobes featured by the casing, the bladed disk can only be solicited an even number of times per revolution which explains that there is no significant response along odd EO lines. Critical speeds correspond to peaks of amplitude in the frequency maps, as for instance around $\Omega \approx 5.1-5.2$. The 2nd, 4th, and 6th EO respond with large amplitudes pictured as blue circles A-C in Fig. 9(a). Figs. 9(b)-(g) depict the nodal diameter contributions over the considered rotational speed range. These frequency maps display the global vibratory behaviour of the impeller when the latter is kinematically restricted to only one of its possible six nodal diameters. In Fig. 9(b)-(g), the aliasing effect is clearly distinguishable. First, the fundamental EO 2 is only visible in Figs. 9(a) and 9(d), which confirms that the impeller response is limited to the 2nd nodal diameter along this specific EO. Similarly, the second super-harmonic EO 4 responds on the 4 th nodal diameter, see Fig. 9(f). Higher harmonics need to be expressed in space using the aliasing effect. The next harmonics EO 6, EO 8, and EO 10 are respectively found along the 4th (Fig. 9(f)), 2nd (Fig. 9(d)), and 0th (Fig. 9(b)) nodal diameters in agreement with Fig. 4.

\section{Configuration 2}

Similarly to the above case the fundamental EO responds dominantly on the 3rd nodal diameter as shown in Fig. 10(a) and 10(e). Next to the fundemental EO, super harmonics of the excitation are clearly visible along the 6 th, 9 th, 12 th, etc. EO. These EO harmonic respond on their corresponding aliased nodal diameters as expected.

Noticeably, non-integer EO responses are also visible in the explored rotational speed range. For example, for $\Omega \approx 4.8$ a major response is present between the 4 th and 5 th EO (see blue circles D and E in Fig. 10 (b) and (d)): regarded in terms of nodal diameter contributions, it cannot be clearly associated to a single nodal diameter, but rather to a combination of $n_{d}=$ 0,1 and 2. This suggests a complex non-integer sub- or super-harmonic contact excitation.

\section{CONCLUSION}

The existing numerical developments devoted to the numerical prediction of undesired unilateral contact induced blade/casing interactions have been extended to full bladed-disk assemblies featuring cyclic symmetry. A wide range of applications may then be foreseen: from the detection of critical speeds of automotive turbochargers under aerodynamic loads to the vibratory response of helicopter engine impellers undergoing contact occurrences. Work is in progress for the detection of critical speeds with both aerodynamical (external forcing) and structural (contact) considerations.

A detailed methodology for the analysis of nodal diameter contributions, subject to a forcing frequency with possible sub and super-harmonics is proposed. By systematically performing two-dimensional Fourier transforms in space and time, the aliasing effect allows for the expression of the fundamental and super-harmonics of contact excitation as nodal diameters. Although minor, sub-harmonic responses are predicted for certain rotational 


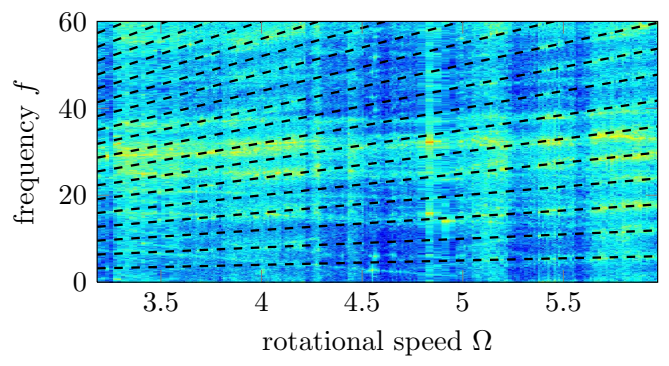

(a) Full Map

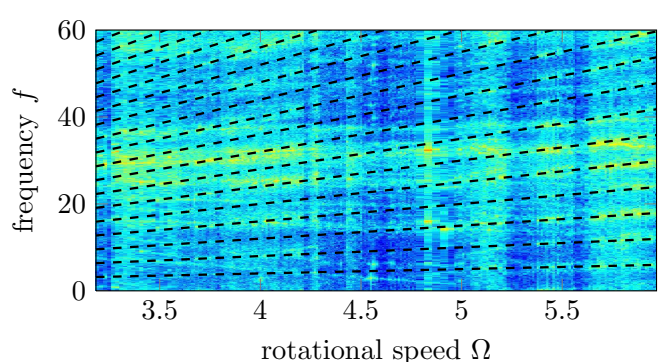

(b) $n_{d}=0$ Component Map

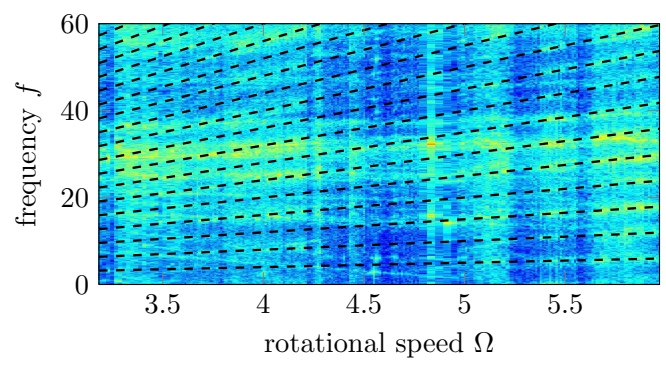

(d) $n_{d}=2$ Component Map

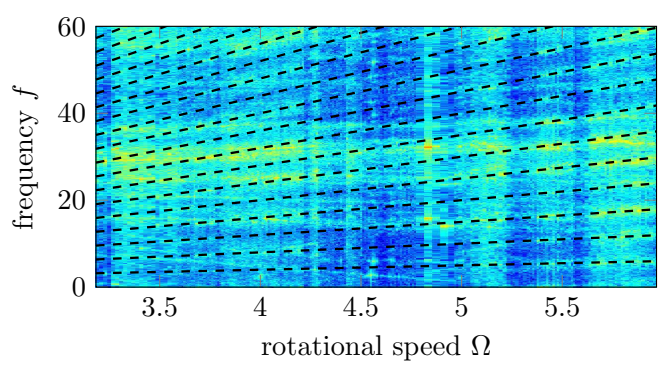

(f) $n_{d}=4$ Component Map

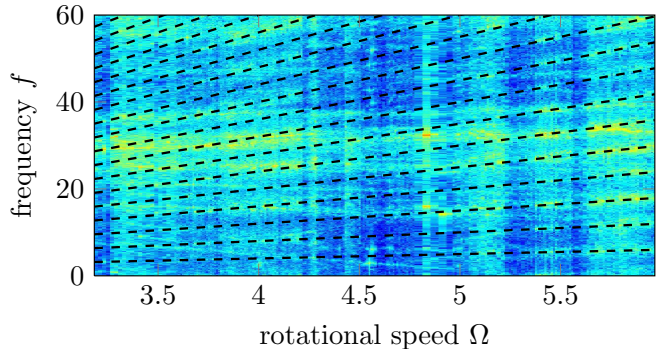

(c) $n_{d}=1$ Component Map

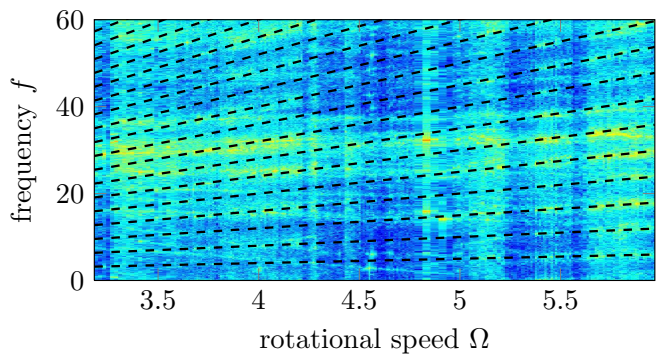

(e) $n_{d}=3$ Component Map

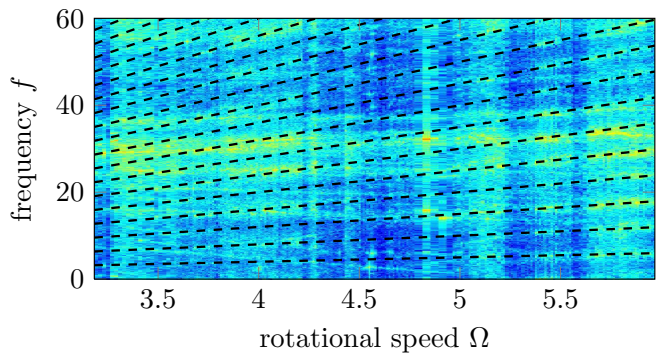

(g) $n_{d}=5$ Component Map

Figure 9 - FREQUENCY MAPS FOR A 2-LOBE CASING DISTORTION 


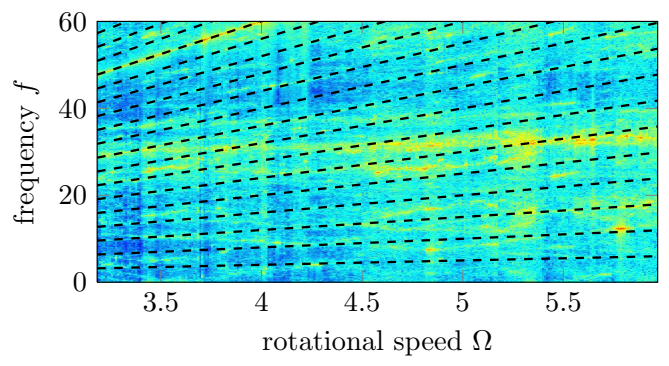

(a) Full Map

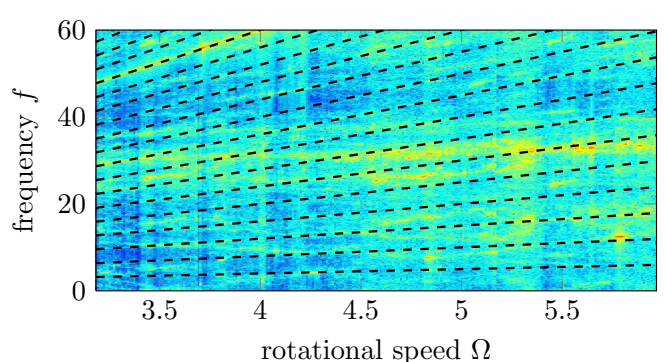

(b) $n_{d}=0$ Component Map

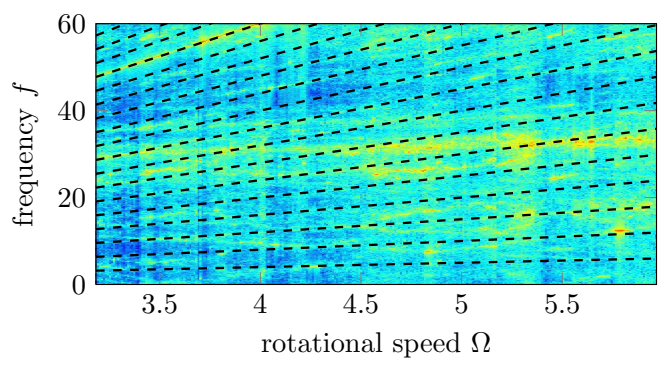

(d) $n_{d}=2$ Component Map

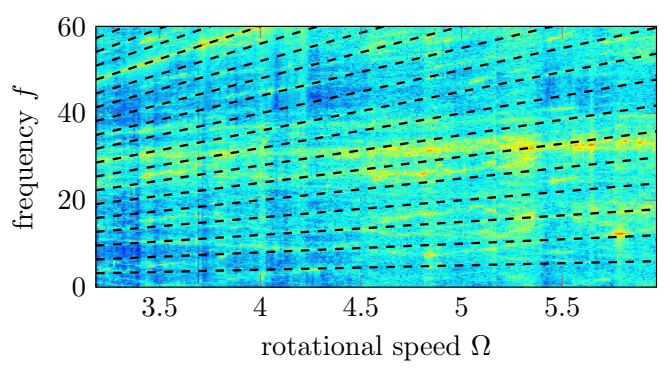

(f) $n_{d}=4$ Component Map

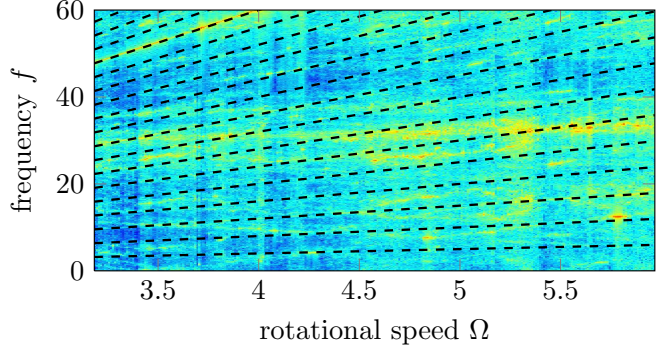

(c) $n_{d}=1$ Component Map

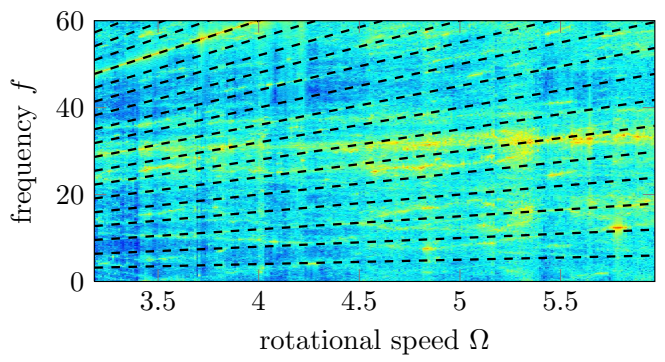

(e) $n_{d}=3$ Component Map

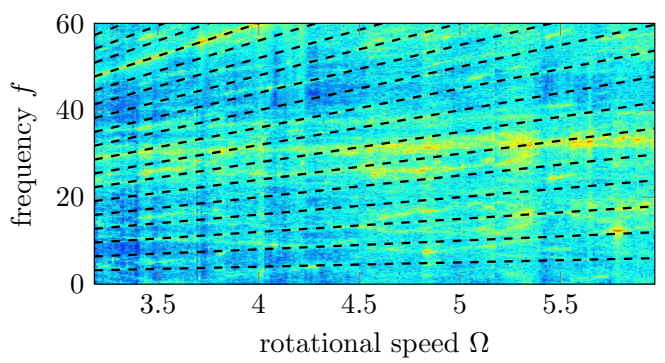

(g) $n_{d}=5$ Component Map

Figure 10 - FREQUENCY MAPS FOR A 3-LOBE CASING DISTORTION 
speeds, that do not comply with the aliasing effect in a straight-forward manner, but are rather originated by a combination of nodal diameter responses.

Further investigations are required to fully determine the contributions of specific modes at any given rotational speed. Effects such as frequency clustering may play a dominant role in the aliasing type response, especially around modal coincidence operating conditions. The response of the cyclic systems as compared to a single blade type setup is not analogous. While a single blade contact geometry introduces super-harmonic content of a specific mode shape, the full cyclic system responds on a super-harmonic of the external forcing on a different nodal diameter, rather than a super-harmonic of the same nodal diameter mode shape.

\section{ACKNOWLEDGEMENT}

Thanks go to Turbomeca and Snecma for their technical and financial support. This work takes place in the framework of the MAIA mechanical research and technology program sponsored by CNRS, ONERA and SAFRAN Group.

\section{References}

[1] Millecamps, A., Brunel, J. F., Dufrénoy, P., Garcin, F., and Nucci, M., 2009. "Influence of thermal effects during blade-casing contact experiments". In Proceedings of the ASME IDETC/CIE. San Diego, USA.

[2] Batailly, A., Legrand, M., Millecamps, A., and Garcin, F., 2012. "Numerical-experimental comparison in the simulation of rotor/stator interaction through blade-tip/abradable coating contact". Journal of Engineering for Gas Turbines and Power, 134(8).

[3] Legrand, M., Batailly, A., Magnain, B., Cartraud, P., and Pierre, C., 2012. "Full threedimensional investigation of structural contact interactions in turbomachines". Journal of Sound and Vibration, 331(11), pp. 2578-2601.

[4] Michimura, S., Nagamatsu, A., and Asazuma, K., 1979. "Vibration of impellers. III- an analysis of coupled vibrations between disk and blades using a reduced impedance method". Bulletin of the JSME, 22(12), pp. 1293-1298.

[5] Michimura, S., Nagamatsu, A., Yamaguchi, H., and Ishikawa, T., 1984. "Vibration of impellers. V- measurement of resonant vibratory stresses of an impeller and pressure distribution due to aerodynamic excitation". Bulletin of the JSME, 27, pp. 534-539.

[6] Michimura, S., Nagamatsu, A., Hagiwara, N., and Kikuchi, K., 1984. "Vibration of impellers. VI- vibration analysis at moving coordinates". Bulletin of the JSME, 27, pp. 19902001.

[7] Michimura, S., Nagamatsu, A., Hagiwara, N., and Kikuchi, K., 1984. "Vibration of impellers. VII- vibration analysis at moving coordinates fixed in rotating impellers". Bulletin of the JSME, 27(231), pp. 1996-2001.

[8] Gibert, C., Blanc, L., Almeida, P., Leblanc, X., Ousty, J.-P., Thouverez, F., and Laîné, J.-P., 2012. "Modal test and analysis of a radial impeller at rest: Influence of surrounding air on damping”. In Proceedings of the ASME Turbo Expo 2012. Copenhagen, Denmark.

[9] Bladh, J. R., 2001. "Efficient predictions of the vibratory response of mistuned bladed disks by reduced order modeling". Phd thesis, University of Michigan.

[10] Thomas, D., 1979. "Dynamics of rotationally periodic structures". International Journal for Numerical Methods in Engineering, 14(1), pp. 81-102. 
[11] Ludeke, C. A., 1942. "Resonance”. Journal of Applied Physics, 13, pp. 418 - 423.

[12] Thompson, J. M. T., and Ghaffari, R., 1982. "Chaos after period-doubling bifurcations in the resonance of an impact oscillator". Physics Letters, 91A, pp. 5-8.

[13] Thompson, J. M. T., and Ghaffari, R., 1983. "Chaos dynamics of an impact oscillator". Physical review, 27, pp. $26-28$.

[14] Bishop, S. R., 1994. "Impact oscillators". Philosophical Transactions: Physical Sciences and Engineering, 347, pp. $347-351$.

[15] Bai, C., Zhang, H., and Xu, Q., 2013. "Subharmonic resonance of a symmetric ball bearing-rotor system”. International Journal of Non-linear Mechanics, 50, pp. 1 - 10. 